\title{
Religione e politica: qualche riflessione sul concetto e sul problema della secolarizzazione e della razionalità nel mondo contemporaneo
}

\author{
Marco Rizzi
}

The paper analyses from an historical point of view the current concepts of "secularization" and "post-secular society". Last decades have shown the failure of the prophecy of the end of the relevance of religion in the public sphere. Far from being a necessary and irreversible trend, secularization - both the European duality between state and church (es) and the American pluralistic "wall of separation" between state and religions - is the historical outcome of the religious wars in 16th century Europa and the self-redefinition of Christianity in front of them. Secularization is thus possible and effective only in presence of religious models grounded on a critical rationality which involves also religious beliefs. Otherwise, the authority principle combined with an instrumental rationality makes secularization ineffective.

Marco Rizzi is Professor of Early Christian Literature at Università Cattolica del Sacro Cuore (Milan).

\section{La persistenza di Dio}

A parere di chi scrive, lo stato attuale del rapporto tra la religione (o le religioni) e la politica (o le istituzioni politico-statuali) può essere compreso solo da una prospettiva storica di medio-lungo periodo e in una articolata visione di geografia culturale, che ridiscuta criticamente la categoria di "secolarizzazione", nonché l'universo concettuale e lessicale ad essa correlato, incluso quello della "postsecolarizzazione" (Berger 1999). Il dato da cui occorre partire è costituito dalla sostanziale smentita delle analisi e delle previsioni elaborate a questo riguardo in ambito storico-culturale e sociologico nel corso della seconda metà del novecento. Tali analisi ritenevano inevitabile il processo di progressiva marginalizzazione dei fattori religiosi nell'ambito della vita pubblica, sia nei suoi termini più direttamente politici, sia per quanto riguardava gli stili di vita individuali e collettivi. Indubbiamente, non veniva esclusa la persistenza delle credenze e della possibilità di adesione alle chiese storiche o ad altre forme di organizzazione collettiva dell'esperienza religiosa, tuttavia se ne ipotizzava una sempre maggiore riduzione alla dimensione esclusivamente privata ed individuale delle persone. In questo modo, si sarebbe definitivamente completato il processo di dissociazione delle 
strutture politiche, sociali e culturali da quelle religiose, avviatosi nell'Europa occidentale nel corso del XVI secolo, o addirittura del precedente (Thiesse 1999).

Iniziatasi con la Riforma, questa dinamica storica ha conosciuto tre significativi momenti di accelerazione che l'avrebbero caratterizzata come irreversibile e destinata a caratterizzare progressivamente l'intera umanità. Dapprima la stagione dell'illuminismo, a sua volta prodotto di un precedente lavorio della storia, ha determinato l'autonomizzazione della ragione umana da ogni dipendenza da un principio di autorità esterno rispetto a essa stessa e alla correttezza del suo procedere, formalizzato sul modello dell'indagine scientifico-matematica: da qui sono nate l'idea di libertà d'espressione e conseguentemente l'opinione pubblica, intesa come forum del dibattito intellettuale che interagisce con la società e la politica nel loro insieme, e risulta la fonte di legittimità per entrambe, sostituendo ogni fondamento religioso.

La rivoluzione americana, prima, e quella francese, poi, sebbene per ragioni profondamente diverse, hanno dato vita a un modello politico corrispondente e parallelo all'emancipazione della ragione dall'autorità religiosa: sotto l'impersonale protezione di un generico teismo, la costruzione politica delle costituzioni americana e francese si edifica dal basso, sulla base dell'uguaglianza delle persone e delle idee, chiamate a confrontarsi e a elaborare proposte di governo in termini di mero confronto razionale intorno alle idea di giustizia, pace, benessere (e non bene) comune, determinando la separazione tra politica e religione.

Infine, la terza accelerazione è stata determinata nel corso del ventesimo secolo e in particolar modo nel secondo dopoguerra dalla diffusione universale di uno stile di vita basato sull'individualismo, sui consumi di beni sempre meno legati alle esigenze primarie, sulla pervasività della scienza e della tecnologia in ogni aspetto della vita. Tutto questo ha determinato nelle società avanzate l'indebolimento dei legami familiari e sociali, il calo demografico, il rovesciamento del rapporto tra tempo destinato al lavoro e tempo dedicato agli interessi individuali, la liberazione dalle faticose routine della vita quotidiana, l'emancipazione femminile, l'emergere di una coscienza giovanile distinta dalla realtà adulta, in sintesi uno stile di vita edonistico - dove il termine va assunto nella sua accezione etimologica, senza alcuna caratterizzazione riduttiva, né tanto meno moralistica (Rémond 2001).

Secondo la più comune interpretazione, questi processi storico-sociali erano destinati ad allargarsi dall'Europa e dalle Americhe (con l'appendice del Giappone) al resto della realtà mondiale. In questo senso è stata originariamente concepita e intesa pure la categoria di globalizzazione, entro cui si ritenevano determinanti e vincenti i processi di "allargamento" dell'occidente rispetto al contro-movimento, soprattutto di tipo migratorio, dai paesi emergenti.

Se singolarmente presi, gli elementi di questa sommaria ricostruzione storica conservano indubbiamente il loro valore ricostruttivo ed euristico. Tuttavia il loro esito complessivo risulta del tutto difforme da quanto ipotizzato da chi li considerava vettori dell'inevitabile processo di marginalizzazione della religione 
nell'orizzonte della società e della politica (Casanova 1994). Non è certo necessario elencare qui tutti gli elementi che, soprattutto a partire dall'11 settembre 2001, hanno portato a revocare in dubbio il valore esplicativo e prognostico del paradigma della secolarizzazione. Basti qui solo ricordare il dato più evidente, anche a livello di cronaca: il ritorno di Dio (o piuttosto la sua mancata scomparsa) quale elemento della discussione e della vita pubblica nelle società europee non è solo il prodotto dei flussi migratori in entrata, bensì anche l'esito di una ridefinizione identitaria in senso religioso di individui nati, cresciuti, educati all'interno di società ad alto grado di apparente secolarizzazione.

\section{Le ambiguità della secolarizzazione: definizioni e indicatori}

Come noto, il concetto di secolarizzazione nasce originariamente in ambito giuridico per indicare la traslazione di un bene dal possesso di un soggetto ecclesiastico a quello di un soggetto di natura non religiosa. Nel lessico storico e sociologico il termine è poi passato a indicare il complesso fenomeno sopra sinteticamente descritto di progressiva riduzione della rilevanza delle esperienze e delle istituzioni religiose all'interno delle società avanzate. Tuttavia, le variazioni degli indicatori di volta in volta utilizzati per meglio individuare questo processo hanno determinato significative oscillazioni e contraddizioni nell'uso e nella comprensione stessa della categoria di "secolarizzazione".

Tipicamente, indicatori fondamentali per valutarne sul piano quantitativo il grado di presenza nella società erano ritenuti la diffusione statistica della credenza in Dio (o in più divinità) e la partecipazione degli individui alle funzioni e alle altre forme di vita religiosa. In termini qualitativi, si cercava di misurare l'incidenza sui comportamenti individuali di valori e insegnamenti riconducibili alla sfera dell'appartenenza religiosa. Si dava un presupposto implicito nelle ricerche basate su questi modelli analitici, prevalentemente condotte nel corso degli anni sessanta e settanta del secolo scorso: la diminuzione degli indicatori quantitativi e qualitativi sarebbe proceduta di pari passo e ciò avrebbe logicamente comportato la conseguente marginalizzazione dell'elemento religioso a livello sociale, politico e culturale. Si sarebbero così diffusi stili di vita sempre meno legati a modelli tradizionali e perciò più "secolarizzati", che a loro volta avrebbero rafforzato il processo di secolarizzazione in atto con un movimento di tipo circolare. In un simile schema interpretativo, "secolarizzazione" risultava sostanzialmente sinonimo di "modernizzazione" e in misura minore di "occidentalizzazione", dato che nella prima categoria rientrava a pieno titolo anche il processo di natura economica, politica e sociale in atto nei paesi comunisti, cui indirettamente si ispirava pure buona parte dei nazionalismi autoritari diffusi in Africa e nel Medio Oriente.

Già nel corso degli anni ottanta e soprattutto nel decennio successivo, questo approccio ermeneutico iniziava a mostrare significative incrinature, segnalate in 
primo luogo dalla rivoluzione del 1978-1979 in Iran, ovvero in un ambito socioculturale in cui il processo di modernizzazione era stato perseguito con estrema determinazione dall'autorità politica (Abrahamian 2008), dall'invasione sovietica dell'Afghanistan dell'anno successivo volta ad impedire la diffusione di analoghe istanze politico religiose nel ventre molle tradizionalmente islamico dell'area caucasica e centro asiatica dell'URSS, ma anche dalla connotazione in senso esplicitamente tradizionalista e cattolico delle proteste operaie in Polonia che portarono alla nascita di Solidarnosc e alla sua successiva messa fuori legge nel 1981 - per limitarsi ai soli fenomeni più vistosi (Kepel 1991).

Al termine del decennio Ottanta, il crollo del muro di Berlino ha di fatto introdotto un ulteriore elemento di distorsione valutativa. Esso è stato considerato una svolta epocale di portata mondiale, sia nei termini più superficiali della sua assunzione quale "fine della storia" (Fukuyama 1992), sia in quelli più storicamente ponderati del "secolo breve" (Hobsbawm 1994). La fine dell'innaturale divisione del continente europeo, la sconfitta del comunismo sovietico provocata dalla supremazia economica e tecnologica delle nazioni occidentali, la prepotente spinta nei paesi dell'ex blocco sovietico per la costruzione della democrazia e dell'economia di mercato, tutto ciò è stato interpretato come se indicasse l'affermazione definitiva e irreversibile non solo di uno schieramento politico-militare, bensì di un modello di società e di cultura civile, quella occidentale, democratica e secolarizzata, appunto.

In realtà, già nel corso degli anni Novanta alcuni tra gli osservatori più attenti e meno legati al mainstream della ricerca e della riflessione socio-culturale avevano iniziato a individuare specifici segnali di differenziazione in questo quadro così apparentemente coerente e monolitico. Se ancora non si giungeva a porre in discussione il paradigma della secolarizzazione, tuttavia si individuava la persistenza e la trasformazione, quando non anche la reviviscenza, delle forme di esperienza e di appartenenza religiosa anche nei contesti apparentemente più legati ai processi di modernizzazione. Particolarmente significative risultavano e risultano le differenze empiricamente riscontrabili tra una concezione "dualista" della secolarizzazione, sostanzialmente riconducibile all'opposizione tra chiesa e stato, e una concezione "pluralista", in cui a venire tematizzato e indagato non è tanto il problema della reciproca delimitazione di ambiti tra il politico e il religioso, quanto la compresenza all'interno della società di uno spettro variegato di opzioni e pratiche di natura religiosa, senza che alcuna di esse possa vantare un posto privilegiato nel rapporto con il sistema politico-statuale. Le due visioni rimandano, rispettivamente, a un modello di secolarizzazione di matrice europea, storicamente inveratosi al suo massimo nella laicité dello stato repubblicano francese, e a uno di impronta statunitense, il cosiddetto "muro di separazione" tra stato e chiese lì vigente (Berger / Davie / Fokas 2008).

Anche in questo caso, però gli schemi ermeneutici più consolidati appaiono non interpretare adeguatamente la realtà di fronte al duplice fenomeno, da un lato, di un ritorno delle identità religiose al ruolo di attori a pieno titolo nell'arena 
politico-sociale e, dall'altro, della moltiplicazione e differenziazione dei soggetti religiosi operanti all'interno di entità politico-statuali che si sono storicamente organizzate nel confronto con un numero più ridotto e tendenzialmente più omogeneo di tradizioni religiose. La pluralità delle chiese, delle sette, delle declinazioni e delle tipologie all'interno di una medesima tradizione è un fenomeno che rende sempre più sfuggente e ambiguo il termine stesso di "religione" per indicare un interlocutore unitario. Una simile differenziazione e frantumazione non è solo il prodotto dei flussi migratori, bensì deriva paradossalmente dall'indebolimento stesso dei soggetti istituzionali, stato e chiese, che sino a tempi recenti organizzavano la vita civile e quella religiosa in una forma dualista o pluralista che fosse.

Di fronte a questa sostanziale aporia che sembra aver imprigionato la categoria di secolarizzazione, sono stati avanzati due tentativi per operarne una ridefinizione, nella convinzione della sua perdurante efficacia, sia in termini descrittivi, sia in termini interpretativi. Si è così provato a costruire più di un nuovo modello ermeneutico che possa superare l'originaria idea di una semplice traslazione di istituzioni e valori dall'ambito del religioso a quello del politico, variando nell'analisi empirica il peso degli indicatori per dimostrare la presenza di comportamenti "moderni" ed individualistici pure in situazioni politiche e culturali caratterizzate da un'intensa presenza pubblica della religione (Norris / Inglehart 2004). Sarebbe ad esempio il caso della società iraniana contemporanea, il cui andamento demografico declinante e la diffusione di stili di vita segnati da influssi occidentali dimostrerebbero l'incidenza della secolarizzazione anche all'interno di quello che risulta essere un vero e proprio stato ierocratico.

Si deve però a Charles Taylor il più ambizioso e recente tentativo di ridefinizione del concetto nei suoi termini storici, filosofici e sociologici. Secondo lo studioso canadese, la secolarizzazione consiste nella progressiva acquisizione di legittimità sociale e culturale di posizioni esplicitamente atee o agnostiche. La politica e lo stato avrebbero progressivamente accompagnato un tale processo storico, giungendo all'odierno atteggiamento di neutralità. Da questa prospettiva, la dimensione religiosa non risulta necessariamente confinata in uno spazio residuale sul piano politico o sociale, ma può giocare legittimamente le sue carte nell'ambito del confronto tra posizioni differenti, senza pretendere alcun ruolo privilegiato, ma neppure risultando un interlocutore culturalmente dimidiato (Taylor 2007). Estremizzando in modo paradossale la prospettiva di Taylor, si potrebbe dire che dopo cinquecento anni la situazione si è rovesciata: l'esito del processo di secolarizzazione è la conservazione della legittimità politica e sociale della credenza in Dio nella società secolarizzata. 


\section{Secolarizzazione o strabismo europeo?}

Di fronte ai paradossi e alle aporie ora evidenziate, ma soprattutto al clamoroso ritorno del fattore religioso quale elemento decisivo per i nuovi equilibri mondiali, è difficile sfuggire all'impressione che i tentativi di ripensamento dell'idea di "secolarizzazione" soffrano di una limitazione prodotta dalla loro elaborazione interna a una prospettiva storico-geografica esclusivamente eurocentrica, quasi che la dinamica innescatasi agli albori dell'epoca moderna nel vecchio continente costituisse il destino ultimo del mondo e dell'umanità intera. $\mathrm{Al}$ contrario, l'esperienza della secolarizzazione così intesa risulta in contrazione, se non addirittura a rischio di concludersi (Micklethwait / Wooldridge 2009; Allawi 2009, per il mondo islamico). Gli avvenimenti susseguenti all'11 settembre e la drammatica accelerazione dei processi di disgregazione delle autorità statuali nel Medio Oriente e nell'Africa settentrionale di questi ultimissimi anni hanno trasferito nell'orizzonte geografico immediatamente a ridosso della culla della secolarizzazione - quand'anche non direttamente al suo interno, come nel caso degli attentati alla metropolitana di Londra o al settimanale parigino Charlie Hebdo - la dimensione inattesa e per questo ancor più sconcertante di processi storico-sociali che solo ora si iniziano a percepire nelle loro reali dimensioni e nella loro effettiva durata e persistenza (Esposito 2002).

Come detto in apertura, la portata della rivoluzione khomeinista è stata a lungo sottostimata nei suoi termini e nelle sue valenze propriamente religiose, che hanno riaperto la strada alla competizione per la leadership religiosa del mondo islamico che non si articola soltanto nella contrapposizione tra sciti, sunniti e altre tradizioni, bensì investe un'area geografica con le sue articolazioni statuali che si estende (almeno) dalla Turchia al Pakistan, dal Kazakhstan al Golfo di Aden. La parabola della Turchia, passata dal laicismo di stato di Atatürk al sogno neoottomano di Erdogan può essere considerata del tutto speculare a quella iraniana, sia pure in un modello cesaropapista anziché ierocratico (Fuller 2008).

Ma anche la "globalizzazione" - per continuare a utilizzare un lessico di comodo - si è rivelata nemica, anziché alleata della secolarizzazione, come invece dai più semplicisticamente ritenuto in un primo tempo (Nanda 2010, per il caso indiano). La diffusione di aspetti dello stile di vita occidentale "moderno" o "secolarizzato" in molte aree culturali e geografiche asiatiche e africane, lungi dall'indebolirle, ha rafforzato e si è combinata con le strutture religiose tradizionali. Gli aspetti più eclatanti vanno dall'uso dei social network per la diffusione della predicazione fondamentalista al consumo individuale di pornografia in contesti sociali e religiosi caratterizzati da un rigido codice di comportamento morale in pubblico, giusto per citare due estremi opposti. Al contrario, il movimento centripeto verso l'Europa dei flussi migratori ha riportato sulla scena del dibattito pubblico europeo problematiche che si ritenevano da tempo pacificamente risolte in senso "secolarizzato", quali la tutela e i confini della libertà di espressione, i limiti della potestà genitoriale in tema di educazione dei 
figli, lo squilibrio nella concreta fruizione dei diritti individuali tra uomini e donne (Kadri 2012) - senza menzionare il riemergere dell'antisemitismo nelle sue forme tradizionali e soprattutto in quelle di matrice islamica.

Dal punto di vista dell'analisi storica e culturale, però, si deve procedere oltre la mera constatazione dell'arretramento, se non addirittura della battuta d'arresto, dei processi di secolarizzazione e di modernizzazione (Graziano 2014). Si può affermare, in termini generali, che al momento e presumibilmente sul mediolungo periodo il problema religioso - o più radicalmente il tema di Dio nelle sue varie declinazioni storiche - continuerà a giocare un ruolo non marginale nelle dinamiche politiche e culturali sia a livello globale, sia a livello locale. Si tratta di un esito legato esclusivamente al declino politico dell'Europa e del fascino del suo modello culturale sul resto del mondo o il fallimento del modello interpretativo della secolarizzazione ha radici più profonde (Ferguson 2012)?

Per quanto paradossale possa sembrare, l'arretramento dei processi di secolarizzazione coincide anche e soprattutto con la crisi del cristianesimo occidentale come modello religioso di riferimento, fatto di istituzioni forti e di un complesso dottrinale organico, ampio e sofisticato. Il modello, per intenderci, in relazione al quale si poteva utilizzare la categoria di "dualismo": dualismo di stato e chiesa, di foro pubblico e foro interiore, di spirituale e secolare, o in termini teologici natura e soprannatura, fini ultimi e fini penultimi, e così via. La molteplicità dei fenomeni religiosi che si stanno affermando in questi ultimi decenni appaiono caratterizzati invece da approcci meno istituzionalizzati ed intellettualizzati, ma più totalizzanti e assorbenti ogni altro aspetto dell'esperienza umana, anche in forme che risultano contraddittorie ad un approccio razionale (Lewis 2002). Si è qui di fronte a un nodo chiave del processo storico che ha portato agli attuali esiti della secolarizzazione europea. Un nodo che l'allora pontefice Benedetto XVI aveva cercato di porre in evidenza nel molto criticato discorso di Ratisbona, ma che è stato occultato dalle polemiche derivate dalla sostanziale impoliticità della sua forma. Tuttavia esso rimane decisivo.

\section{Secolarizzazione, razionalità, cristianesimo}

Sin dalle origini, il cristianesimo ha istituito un rapporto costitutivo con un modello di razionalità, quello della speculazione filosofica ellenistica, che ne ha sostanziato il processo di progressiva autodefinizione e di elaborazione delle sue strutture teologiche. In questo modo, il cristianesimo ha inglobato al proprio interno un'istanza critica e autocritica (nel senso della riflessione sul proprio statuto) che ha assegnato una funzione fondativa e ineliminabile alla razionalità, che non può in alcun modo risultare meramente strumentale $\mathrm{o}$ al contrario del tutto separata rispetto a questo orizzonte religioso. Non solo, ma in precedenza, nel processo di confronto e distacco dall'originaria matrice giudaica, il cristianesimo si era venuto caratterizzando per l'intrinseca dimensione ermeneutica, 
scaturita dalla necessità di elaborare una propria, differente interpretazione del testo sacro condiviso, la Bibbia. Tale specificità ermeneutica riguardava anzitutto il problema della legge (mosaica), non semplicemente in termini di interpretazione del singolo dettato normativo, bensì del suo significato complessivo alla luce di un principio estrinseco ad essa, la fede in Gesù Cristo quale suo ultimo compimento. Sintetizzando al massimo complessi processi storici, si può dire che è sempre stata operativa nell'esperienza cristiana una razionalità critica ed ermeneutica, necessariamente combinata con un elemento di autorità costituito dalla rivelazione nelle sue diverse forme, la Bibbia-legge e Cristo-logos.

Si sono così create le condizioni perché anche in una società largamente caratterizzata dall'elemento religioso l'individuo potesse elaborare la propria soggettività sulla base di una razionalità comune e condivisa con le istituzioni religiose, che non potevano limitarsi alla semplice evocazione del principio di autorità per legittimare la propria azione. Analogamente, una volta innescatosi il processo di secolarizzazione, le istituzioni civili hanno trovato nelle varianti del cristianesimo europeo un interlocutore spesso conflittuale, ma sostanzialmente compatibile, in quanto entrambi provenienti in ultima analisi dalla medesima matrice (Audi 2011).

Ciò non vuol dire affatto che la secolarizzazione o la separazione tra chiesa e stato sia un prodotto diretto del cristianesimo, come una maldestra apologetica contemporanea vorrebbe far credere. Semplicemente, significa che quella che chiamiamo "secolarizzazione" è stata possibile in presenza di una specifica struttura teologica che, in altrettanto specifiche circostanze storiche, ha favorito l'emersione e la legittimità di una razionalità autonoma, non subordinata né totalmente separata rispetto alla religione. Anche in questo caso si tratta di un processo storico di lungo periodo, in cui è risultato decisivo l'impatto delle guerre di religione del XVI secolo, non a caso comunemente posto quale momento d'avvio della modernità e del processo di secolarizzazione.

Nel corso del secolo e più che va dall'avvio della Riforma protestante alla pace di Vestfalia, la spinta decisiva degli avvenimenti per superare i conflitti religiosi è stata accompagnata e indirizzata dalla riflessione interna alle diverse denominazioni cristiane, che pur partendo da un aspetto specifico ha finito per investire anche elementi fondativi della concezione di Dio e del suo rapporto con l'uomo e il mondo. Si è così prodotto un mutamento di ordine teologico delle categorie entro cui si strutturavano le esperienze e le istituzioni religiose nella società. Basti pensare, sul versante cattolico, all'elaborazione della dottrina bellarminiana della potestas indirecta, che ha di fatto posto fine alla pretesa papale di un esercizio diretto dell'autorità spirituale in saecularibus, sin lì una cifra caratterizzante, per quanto dibattuta, del cristianesimo medievale. $\mathrm{O}$, sul versante riformato, al rovesciamento nel calvinismo dei Paesi Bassi del rapporto tra leadership ecclesiale e leadership civile, passaggio imprescindibile per comprendere il successivo sviluppo puritano che ha portato al "muro di separazione" tra chiesa e 
stato negli USA. In tutti questi casi, cambiamento storico e riflessione teologica hanno proceduto di pari passo, in un rapporto circolare di causa-effetto.

Occorre ribadire ancora una volta, a scanso di equivoci, che dal punto di vista storico questo non va inteso come un esito necessitato, bensì come il prodotto di caratteri teologici e contingenze storiche. Per fare ancora una volta l'esempio dell'islam, non è che siano mancati anche nella sua storia tentativi di marca razionalistica, da Al Farabi ad Averroé, per citare i più celebri. Semplicemente o le condizioni storiche, o le strutture teologiche allora dominanti, o la combinazione di entrambe non hanno permesso i medesimi esiti osservati per il cristianesimo occidentale.

Se così è, ne consegue che il paradigma della secolarizzazione come sin qui inteso non ha valore assoluto, ma vale storicamente e spiega solo una determinata fase della storia del cristianesimo che potenzialmente può coincidere con la sua estinzione - ma non è detto che ciò debba necessariamente avvenire. Comune a tutti i teorici della secolarizzazione, infatti, risulta essere la fiducia nella funzione della razionalità individuale quale cifra determinante dei processi sociali grazie a cui anche i fenomeni di integrismo o di estremismo religioso risultavano destinati, se non a scomparire, a trovare comunque forme di assestamento e di compatibilità all'interno delle società moderne, come evidenziato nel caso dei movimenti carismatici o evangelicali del nord e del sud America. Il problema si pone invece in termini del tutto differenti - e l'idea di secolarizzazione diventa uno strumento analitico inefficace - quando l'esperienza religiosa risulta disgiunta da un modello di razionalità critica e sottoposta invece ad un principio di autorità, che si combina con il solo aspetto strumentale della ragione in un modello che sembra contrassegnare oggi il prepotente ritorno della religione sulla scena pubblica mondiale.

\section{Teologia e trasformazioni religiose}

Se il paradosso sopra indicato per cui la crisi della secolarizzazione coincide con la crisi del primato del modello religioso cristiano esprime una situazione reale, se ne debbono trarre due conseguenze di rilievo, a parere di chi scrive.

La prima è che le società occidentali "secolarizzate" non possono affrontare il rapporto con i fenomeni religiosi emergenti al loro interno e sullo scenario mondiale - non solo l'islam, bensì anche le varie forme di fondamentalismo cristiano o ebraico (Ali 2002) - allo stesso modo e con gli stessi strumenti con cui lo hanno fatto e lo fanno con le chiese cristiane tradizionali o con le altre denominazioni religiose che accettano di adeguarsi a quel modello. Ad esempio, il mantra della laicité francese ("a casa ebrei, musulmani, cristiani, a scuola solo francesi") può funzionare solo con chi è disposto ad accettare una dimensione religiosa che contempli la possibilità di accettare e razionalizzare una forma di dualismo, rispetto ad una pretesa veritativa assoluta. Se nella scuola si insegna la 
separazione, ma non si favorisce la conoscenza e la riflessione critica su di sé e sulla propria tradizione religiosa il dualismo resta ingiustificato e privo di credibilità.

La seconda conseguenza rilevante è che neppure la diffusione di benessere, scienza e tecnologia comporta necessariamente l'abbandono o l'incompatibilità delle credenze religiose. Il combinato disposto di un modello religioso basato sul principio d'autorità (del libro, della rivelazione o del guru non risulta a questo proposito particolarmente rilevante) e di una concezione meramente strumentale, non critica della razionalità rende possibile e del tutto coerente provenire da una buona famiglia, studiare, diventare ingegneri e schiantarsi contro un grattacielo alla guida di un aereo dirottato gridando il nome di Dio - cosa che apparirebbe quantomeno contraddittoria ad un prodotto tipico dell'École normale superieure e della visione del mondo che l'ispira.

Se dunque "Dio" è tornato a essere un tema non più omissibile nella comprensione e nel possibile governo delle attuali dinamiche storiche, a livello globale e locale, c'è da chiedersi quali possano essere gli strumenti di analisi che meglio possano contribuire a questi fini. Per quanto possa sembrare anacronistico, che lo si voglia o no è la "teologia" - intesa come discorso critico sulla propria e altrui tradizione religiosa - ad assumere una centralità decisiva, al di la delle differenziazioni formali dell'oggetto. Paradossalmente, più degli intellettuali europei sembrano averlo capito i leader di alcuni stati arabi direttamente sottoposti alle tensioni generate dall'espansione dell'islamismo radicale, nel momento in cui sollecitano alle autorità religiose dei rispettivi paesi una presa di posizione che neutralizzi sul piano della legittimità religiosa - ovvero della teologia in senso lato - l'interpretazione dell'islam che sta alla base di quei movimenti. Si dovrebbe trattare di una presa di posizione che non si limiti all'enunciazione di un vincolo esterno, quale potrebbe essere l'equilibrio politico locale o internazionale, bensì si esprima nelle categorie proprie dell'elaborazione del discorso religioso, ne ripensi i contenuti di fondo e non discetti semplicemente dell'interpretazione di questo o quel versetto coranico, di questo o di quel detto del Profeta.

Con l'occhio dello storico, è suggestivo osservare come ciò appaia, nell'attuale situazione di conflitto intra-islamico e di crisi delle autorità statuali di gran parte del mondo arabo, un tentativo di riprodurre la dinamica innescata dalle guerre di religione nel cinquecento europeo. Non è dato sapere se un simile processo potrà produrre esiti analoghi nel laboratorio mediorientale, se ne possa nascere una secolarizzazione islamica, quanto e come quella tradizione religiosa ne possa risultare trasformata. Allargando il discorso al complesso dei fenomeni in atto sulla scala globale, quello che appare comunque certo è che il discorso su Dio è tornato a occupare un ruolo centrale nel mondo contemporaneo, non può più essere liquidato come semplice residuo di un passato premoderno destinato a scomparire sotto l'avanzata della scienza, della tecnologia e della "secolarizza- 
zione", e necessita di strumenti e interpreti all'altezza di un compito in gran parte inatteso o non per tempo compreso.

\section{Bibliografia}

Abrahamian, Ervand: A History of Modern Iran. Cambridge 2008.

Ali, Tariq: The Clash of Fundamentalism. Crusades, Jiahds, and Modernity. London 2002.

Allawi, Ali A.: The Crisis of Islamic Civilization. New Haven 2009.

Audi, Robert: Rationality and religious commitment. Oxford 2011.

Berger, Peter: The Desecularization of the World. Resurgent Religion and World Politics. Michigan 1999.

Berger, Peter / Davie, Grace / Fokas, Effie: Religious America, Secular Europe? A Theme and Variations. Aldershot 2008.

Casanova, José: Public Religions in the Modern World. Chicago 1994.

Esposito, John L.: Unholy Wars. Terror in the Name of Islam. Oxford 2002.

Ferguson, Niall: Civilization. The West and the Rest. New York 2012.

Fukuyama, Francis: The End of History and the Last Man. New York 1992.

Fuller, Graham E.: The New Turkish republic. Turkey as a Pivotal State in the Muslim World. Washington 2008.

Graziano, Manlio: Guerra santa e santa alleanza. Religioni e disordine internazionale nel XXI secolo. Bologna 2014.

Hobsbawm, Eric: Age of Extremes. The Short Twentieth Century. London 1994.

Kadri, Sadakat: Heaven on earth. A Journey through Sharia Law from the Desert of Ancient Arabia to the Streets of the Modern Muslim World. New York 2012.

Kepel, Gilles: La revanche de Dieu. Chrétiens, juifs et musulmans à la reconquête du monde. Paris 1991.

Lewis, Bernard: What Went Wrong? The Clash between Islam and Modernity in the Middle East. Oxford 2002.

Micklethwait, John / Wooldridge, Adrian: God is back. How the Global Revival of Faith is Changing the World. New York 2009.

Nanda, Meere: The God Market. How Globalization is Making India more Hindu. New York 2010.

Norris, Pippa / Inglehart, Ronald: Sacred and Secular. Religion and Politics Worldwide. Cambridge 2004.

Rémond, René : Religion et société en Europe. Essai sur la sécularisation des sociétés européennes aux XIX ${ }^{\mathrm{e}}$ et $\mathrm{XX}^{\mathrm{e}}$ siècles (1789-2000). Paris 2001.

Taylor, Charles: A Secular Age. Cambridge 2007.

Thiesse, Anne-Marie: La création des identités nationales. Europe XVIII ${ }^{\mathrm{e}}-\mathrm{XX}^{\mathrm{e}}$ seècle. Paris 1999. 